SHORT REPORT

\title{
Application of variable life adjusted display (VLAD) in early detection of deficiency in trauma care
}

\author{
H B Tan, S F Cross, S W Goodacre
}

Emerg Med J 2005;22:726-728. doi: 10.1136/emj.2004.017277

Early detection of deficient care is an increasingly important element of trauma audit. We aimed to assess the feasibility and demonstrate the use of a variable life adjusted display (VLAD) in trauma audit. Data from the Trauma Audit and Research Network database of Sheffield Teaching Hospitals NHS Trust were used to create a VLAD. A cumulative display of survival was plotted in which survivors were incorporated as a positive value equal to 1 minus the probability of survival, and deaths were incorporated as a negative value equal to the probability of survival. Downward deflections of the display thus indicated potentially deficient trauma care. Data from 191 consecutive patients over 1 year were plotted and displayed. The first 2 months of this period were characterised by a downward trend in the line, which may indicate suboptimum performance and provides an example of a trend that would prompt detailed review. The VLAD chart is a potentially useful "early warning" system for poor performance in trauma care. Further work should to be carried out to evaluate VLAD prospectively as an audit tool, perhaps involving comparison of VLAD charts from different institutions.

$\mathrm{V}$ arious methods have been suggested to improve the quality of trauma care and subsequently survival. The Injury Severity Score (ISS) ${ }^{1}$ and the Trauma and Injury Severity Score (TRISS) ${ }^{2}$ are commonly used predictors of survival, alongside databases such as the Trauma Audit Research Network (TARN) ${ }^{3}$ in the UK, and registries in the USA such as the New York State Trauma Registry ${ }^{4}$ and the National Trauma Data Bank. ${ }^{5}$ These methods and databases are used retrospectively to measure the quality of trauma care, but as they require collection of a considerable number of data before analysis can be carried out, a prolonged time may lapse before any discrepancy or poor quality of care is found and rectified.

Recently, a method of early detection for variation in clinical practice has been suggested for acute myocardial infarction $^{6}$ and cardiac surgery. ${ }^{7}$ The variable life adjusted display (VLAD) is a graphical technique based on the cumulative sum method that incorporates information of estimated risks for each individual case. This allows early identification of variations in patient outcome, which is not revealed by block audit. ${ }^{6}$ We believe that this method can be applied for use in trauma care, and this study aimed to assess its feasibility and demonstrate its use in trauma audit.

\section{PATIENTS AND METHODS}

Data were derived from the existing TARN database of the Sheffield Teaching Hospitals. The Sheffield Teaching Hospitals has its emergency department (ED) on two sites; an adult ED based at Northern General Hospital and a paediatric ED at Sheffield Children's Hospital. Together, these two sites provide emergency care to a population of 530 000. TARN was set up in the late 1980s to "collect and analyse clinical and epidemiological data and thereby provide a statistical base to support clinical audit, aid the development of trauma services, and inform the research agenda". ${ }^{3}$ In the Sheffield Teaching Hospitals, a dedicated audit officer was appointed in 1989 to collect TARN data. Inclusion and exclusion criteria for data collection are illustrated in table 1. Data collected were collated using Microsoft Access software and analysed using Microsoft Excel.

We analysed all patients who were entered into the TARN database from 3 January to 30 December 2001. Demographic details of patients included age, sex, date and time of injury, type of injury, Glasgow Coma Score, emergency procedures and investigations carried out, length of stay, diagnosis, grade and specialty of attending doctors, and outcome. Diagnosis was coded according to the Abbreviated Injury Scale. ${ }^{8}$ Probability of survival $(\mathrm{x})$ was calculated according to the TRISS methodology, based on Injury Severity Score (ISS), Revised Trauma Score (RTS), and age code (table 2). Patients who survived were given a score of 1 minus their probability of survival $(1-x)$, while patients who died were given a negative value equal to their probability of survival $(-x)$. Thus, a patient with high probability of survival who survived attracted a low positive score, whereas if the same patient died, a high negative score would be obtained. A cumulative survival score was obtained by serial summations of these scores chronologically. A graph of cumulative survival over time is thus obtained from this result. Downward deviations in the graph line may represent suboptimum performance and are thus used to prompt detailed audit and review. We subjectively reviewed this graph for any falls indicating potential suboptimum performance.

We used a Microsoft Excel spreadsheet to calculate and plot cumulative survival. Each row contained details for a new patient. Column A indicated whether the patient survived (Live) or died (Death), and column B contained the probability of survival. The formula = IF $(\mathrm{A} \#=$ "LIVE", $\mathrm{l}-\mathrm{B} \#,-\mathrm{B} \#$ was inserted in column $\mathrm{C}$ (with \# being substituted for the relevant row number). Column D sequentially added the contents of column $C$ to each new row to calculate cumulative survival. The VLAD was thus a plot of column D against time.

\section{RESULTS}

In total, 191 patients were analysed, of whom 26 died. The mean ISS score was 14.6. There were 126 males and 65 females. The mean length of stay in hospital was 17.1 days,

Abbreviations: ED, emergency department; ISS, Injury Severity Score; NATD, National Trauma Data Bank; RTS, Revised Trauma Score; TARN, Trauma Audit and Research Network; TRISS, Trauma and Injury Severity Score; VLAD, variable life adjusted display 
Table 1 Criteria for inclusion and exclusion into the TARN database ${ }^{10}$

All trauma patients irrespective of age who meet any of the following
criteria:
All trauma admissions whose length of stay is 72 hours or more
All trauma patients admitted to an intensive care or high dependency
area
All deaths of injured patients occurring in the hospital (even if the cause
of death is medical)
All trauma patients transferred to another hospital for further
emergency care or admission to a high dependency area or die from
injuries
Patients with the following conditions were excluded:
Isolated fractures of the femoral neck or single pubic rami fractures in
patients aged $\geqslant 65$ years
Or isolated closed, undisplaced, not comminuted limb injuries
Or soft tissue spinal strains
Or closed, undisplaced facial injuries
Or simple penetrating injuries not involving deeper structures or $<20 \%$
blood loss
And/or uncomplicated skin injuries
And/or I $>10 \%$ superficial or partial thickness burns (all full thickness
burns are included)

with $7.85 \%$ needing admission into an intensive or high dependency care unit.

The cumulative probability of survival score obtained was plotted against time (fig 3 ). The first 2 months of this period are characterised by a downward trend in the line that may indicate suboptimum performance. Cases managed during this period would thus be selected for detailed review. As this latter process involves compromise of patient confidentiality, it could only be undertaken as part of an in hospital audit process or an approved independent review.

\section{DISCUSSION}

VLAD charts are a potentially useful tool for trauma audit because of their ability to pick up downward trends in performance at an early stage. With increasing pressure upon institutions to monitor and demonstrate good performance, there is a growing importance to develop such earlydetection mechanisms. In 1995, there was a scandal
Table 2 TRISS calculation of probability of survival

Injury Severity Score (ISS)

The sum of the squares of the largest AIS values assigned to the three most severe injuries in different body regions. Unless patient has AIS value of 6 , regardless of other injuries sustained, they are assigned an ISS of 75

Revised Trauma Score (RTS)

The sum of the weighted scores of blood pressure, Glasgow Coma Scale, and respiratory rate

Age code

Age $<55$ years $=0$

Age $>55$ years $=1$

Probability of survival (PS)

$P S=1 /(a+e-b)$ where $b=b 0+b 1$ (RTS) + b2 (ISS) + b3 (AC) Constants $\mathrm{bO}-3$ and weighting factors applied to these variables are derived from analysis of large numbers of patients in the major trauma outcome study

concerning substandard paediatric cardiac surgery in Bristol. ${ }^{9}$ Healthcare professionals within the Bristol unit had voiced concerns since the late 1980s, but it was only in 1995, with the death of a paediatric patient on the operating table, that an enquiry was undertaken and appropriate action taken. ${ }^{9}$ VLAD charts provide an objective means of monitoring performance that can be used to prompt more detailed audit when poor performance is suspected. Previous publications has suggested VLAD to be useful in monitoring surgical performance in cardiac surgery, ${ }^{7}$ as well as in care of patients with acute myocardial infarction. ${ }^{6}$

Care must be taken in the interpretation of a VLAD chart. The simplicity of this system could lead to false positives, where the "dips" in VLAD may be due to random error, and not a reflection of a deficiency in quality of care. The all or nothing measure (alive or dead) will not detect deficiencies in quality of care that do not lead to death. Furthermore, the usefulness of this system for early prediction is limited by the rate of data collection. In our institution, TARN data are collected by a trauma audit officer who from 2000 is only employed to work 15 hours a week. This leads to a time delay in data collection, as well as the possibility of inadvertent missing or incomplete data collection, owing to time constraints.

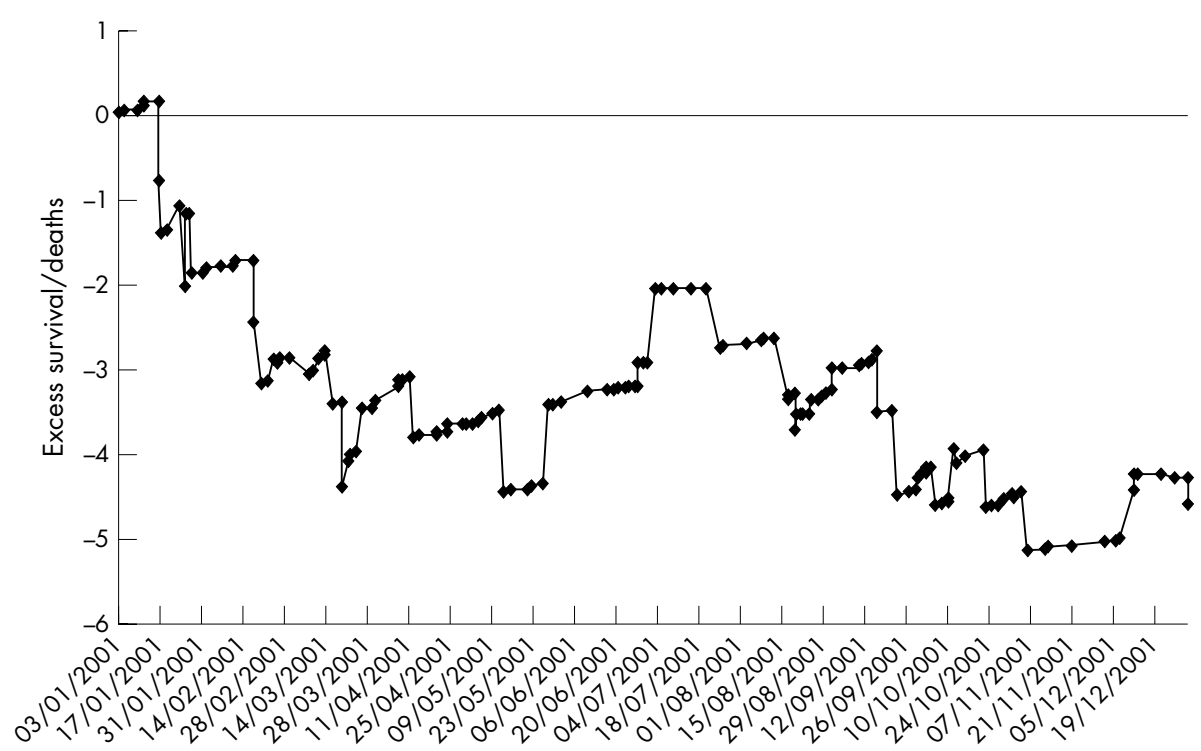

Figure 3 Variable life adjusted display of trauma mortality, NGH 2001. 


\section{CONCLUSION}

The VLAD chart is a potentially useful "early warning" system for poor performance in trauma care. Further work should to be done to evaluate VLAD prospectively as an audit tool, perhaps involving comparison of VLAD charts from different institutions.

\section{Authors' affiliations}

H B Tan, S F Cross, S W Goodacre, Emergency Department, Northern General Hospital, Herries Road, Sheffield, UK

Competing interests: none declared

Correspondence to: Dr S W Goodacre, Senior Lecturer and Consultant Department of Accident and Emergency, Northern General Hospital, Herries Road, Sheffield, S5 7AU, UK; s.goodacre@sheffield.ac.uk

Accepted for publication 9 August 2004

\section{REFERENCES}

1 Baker SP, O'Neill B, Haddon W, et al. The injury severity score: a method for describing patients with multiple injuries and evaluating emergency care. J Trauma 1974;14:187-96.

2 Boyd CR, Tolson MA, Copes WS. Evaluating trauma care: the TRISS method. Trauma Score and the Injury Severity Score. J Trauma 1987;27:370-8.

3 The UK Trauma Audit and Research Network. www.tarn.ac.uk/main.asp.

4 New York State Department of Health. New York State Trauma Program. www.health.state.ny.us/nysdoh/ems/nystrauma.htm.

5 National Trauma Data Bank (NTDB). www. facs.org/trauma/ntdb.html.

6 Lawrence RA, Dorsch MF, Sapsford RJ, et al. Use of cumulative mortality data in patients with acute myocardial infarction for early detection of variation in clinical practice: observational study. BMJ 2001;323:324-7.

7 Sherlaw-Johnson C Lovegrove J, Treasure T, et al. Likely variations in perioperative mortality associated with cardiac surgery: when does high mortality reflect bad practice? Heart 2000;84:79-82.

8 Association for the Advancement of Automotive Medicine. The abbreviated injury scale, 1990 revision. Des Plaines: Association for the Advancement of Automotive Medicine, 1990.

9 The Bristol Royal Infirmary Inquiry. Learning from Bristol: the report of the public inquiry into children's heart surgery at the Bristol Royal Infirmary 19841995. www.bristol-inquiry.org.uk/index.htm.

10 National Trauma Audit and Research Network. Developing effective care for injured patients through outcome analysis and dissemination: operational guidelines. Salford: The UK Trauma Audit and Research Network, 1998. 